\title{
VISCOUS PROFILES OF TRAVELING WAVES IN SCALAR BALANCE LAWS: THE CANARD CASE *
}

\section{J. HÄRTERICH ${ }^{\dagger}$}

\begin{abstract}
The traveling wave problem for a viscous conservation law with a nonlinear source term leads to a singularly perturbed problem which necessarily involves a non-hyperbolic point. The correponding slow-fast system indicates the existence of canard solutions which follow both stable and unstable parts of the slow manifold.

In the present paper we show that for the viscous equation there exist such heteroclinic waves of canard type. Moreover, we determine their wave speed up to first order in the small viscosity parameter by a Melnikov-like calculation after a blow-up near the non-hyperbolic point. It is also shown that there are discontinuous waves of the inviscid equation which do not have a counterpart in the viscous case.
\end{abstract}

1. Introduction. In [Här00], we started the study of viscous profiles of scalar hyperbolic balance laws

$$
u_{t}+f(u)_{x}=g(u), \quad x \in \mathbb{R}, \quad u \in \mathbb{R}, \quad f \in C^{3}, \quad g \in C^{2} .
$$

Equations of this type are often considered as an approximation for a viscous equation

$$
u_{t}+f(u)_{x}=\varepsilon u_{x x}+g(u), \quad x \in \mathbb{R}, \quad u \in \mathbb{R}
$$

where the viscosity parameter $\varepsilon$ is very small. Applications of balance laws, although not scalar, can be found e.g. in combustion [PC86], nozzle flow [CG96] and describing roll waves [NT01].

Our main interest in this paper are continuous traveling wave solutions since they are a feature that distinguishes hyperbolic balance laws from the much better studied hyperbolic conservation laws.

In the hyperbolic case $\varepsilon=0$, Mascia [Mas97] has given a classification of the possible traveling waves for the case of a convex flow function $f$. Here we will treat the question whether these traveling waves are admissible with respect to the viscosity criterion, i.e. whether they can be obtained as limits of traveling waves of the viscous balance law (2) when $\varepsilon$ tends to zero. We are also interested in the influence of the small viscosity on the speed of the traveling wave. Estimates on this wave speed correction are necessary to prove the convergence of the wave profiles in $L^{1}(\mathbb{R})$ for $\varepsilon \rightarrow 0$. It turns out that not all solutions are viscosity solutions in this sense. In particular, all waves of the hyperbolic equation with more than one discontinuity do not admit viscous profiles.

We assume the following about the nonlinear functions $f$ and $g$ :

(G) $g$ possesses exactly three simple zeros $u_{\ell}<u_{m}<u_{r}$ with $u^{\prime}\left(u_{\ell}\right)<0, u^{\prime}\left(u_{m}\right)>$ 0 and $u^{\prime}\left(u_{r}\right)<0$.

(F) $f^{\prime \prime}\left(u_{m}\right)>0$ and $f^{\prime}\left(w_{1}\right)<f^{\prime}\left(u_{m}\right)<f^{\prime}\left(w_{2}\right)$ for all $w_{1}<u_{m}<w_{2}$.

Note that for the pure reaction dynamics $u_{t}=g(u)$ the zeroes $u_{\ell}$ and $u_{r}$ are attracting while the zero $u_{m}$ is repelling.

Since the hyperbolic equation (1) does in general not possess global smooth solutions, and since on the other hand weak solutions are not unique, one considers

\footnotetext{
*Received August 29, 2002; accepted for publication July 7, 2003.

${ }^{\dagger}$ Department of Mathematics, Free University Berlin, Arnimallee 2-6, 14195 Berlin, Germany (haerter@math.fu-berlin.de).
} 
as a solution usually a weak solution which satisfies an additional so-called entropy condition. As this paper is concerned with traveling waves only, we state directly for the special case of traveling waves what is meant by a (possibly discontinuous) entropy solution of (1) .

DEFINITION 1.1. An entropy traveling wave is a solution of (1) of the form $u(x, t)=u(x-s t)$ for some velocity $s \in \mathbb{R}$ with the following properties:

(i) $u$ is piecewise $C^{1}$, i.e. $u \in C^{1}(\mathbb{R} \backslash J)$ and the set of accumulation points of $J$ has only isolated points. At points where $u$ is continuously differentiable it satisfies the ordinary differential equation

$$
\left(f^{\prime}(u(\xi))-s\right) u^{\prime}(\xi)=g(u(\xi))
$$

with $\xi=x-$ st.

(ii) The jumps at the points of discontinuity satisfy the Rankine-Hugoniot condition

$$
s(u(\xi+)-u(\xi-))=f(u(\xi+))-f(u(\xi-))
$$

and the entropy condition

$$
u(\xi+) \leq u(\xi-)
$$

where $u(\xi+)$ and $u(\xi-)$ stand for the one-sided limits of $u$.

1.1. Heteroclinic waves of the hyperbolic equation. In this paper we are primarily interested in traveling wave solutions which connect constant states at $\pm \infty$.

Definition 1.2. A traveling wave $u$ is said to be a heteroclinic wave connecting equilibria $u_{-\infty}$ and $u_{+\infty}$ if

$$
\lim _{\xi \rightarrow-\infty} u(\xi)=u_{-\infty}, \quad \lim _{\xi \rightarrow+\infty} u(\xi)=u_{+\infty}
$$

for some $u_{-\infty}, u_{+\infty} \in \mathbb{R}$ with $u_{-\infty} \neq u_{+\infty}$.

It is called a homoclinic wave if $u_{-\infty}=u_{+\infty}$.

Apart from the heteroclinic waves there can be also discontinuous periodic and nonperiodic waves.

There are several types of heteroclinic waves as shown in [Mas97]. They fall into three categories:

(A) Heteroclinic waves which exist for a whole interval of wave speeds $s$

(B) waves which can be found only if the speed $s$ takes precisely the value $f^{\prime}\left(u_{m}\right)$ and

(C) undercompressive shock waves which do also show up only for isolated wave speeds determined by a Rankine-Hugoniot condition.

In the present paper we are mainly interested in the waves of type (B). A finer classification allows to split these waves into four subcategories (B1)-(B4), see [Här00]. However, under assumption $(\mathbf{G})$ only two cases can actually occur:

(B1) Continuous, monotone increasing waves connecting $u_{\ell}$ to $u_{r}$ with speed $s=$ $f^{\prime}\left(u_{m}\right)$

(B4) Discontinuous waves that connect $u_{\ell}$ to $u_{r}$ with speed $s=f^{\prime}\left(u_{m}\right)$. 
Note that for $s_{0}:=f^{\prime}\left(u_{m}\right)$ the traveling wave equation (3) can be put in the form

$$
u^{\prime}= \begin{cases}\frac{g(u)}{f^{\prime}(u)-f^{\prime}\left(u_{m}\right)} & \text { for } u(\xi) \neq u_{m} \\ \frac{g^{\prime}\left(u_{m}\right)}{f^{\prime \prime}\left(u_{m}\right)} & \text { for } u(\xi)=u_{m}\end{cases}
$$

The wave speed $s_{0}$ is the only one for which the singularity of the function $g(u)\left(f^{\prime}(u)-s\right)^{-1}$ can be removed. Therefore, there exists a monotone heteroclinic orbit $u_{0}$ from $u_{\ell}$ to $u_{r}$ precisely for this wave speed. Considered as a traveling wave, $u_{0}$ is a continuous wave of type (B1). There are also entropy traveling waves with an arbitrary number of discontinuities. They consist of smooth parts which are solutions of (5) separated by discontinuities which connect a left state from the interval $\left(u_{m}, u_{r}\right)$ to the corresponding right state in the interval $\left(u_{\ell}, u_{m}\right)$ according to the Rankine-Hugoniot condition (4). These waves are all of type (B4). Note that these waves of type (B4) pass at least twice (continuously) through the value $u_{m}$. This fact will be used to show that these entropy traveling waves have no counterpart in the viscous equation. In contrast, it will turn out that in the viscous equation only a monotone heteroclinic orbit connecting the equilibria $u_{\ell}$ and $u_{r}$ exists for exactly one wave speed $s(\varepsilon)$ close to but not equal to $s_{0}=f^{\prime}\left(u_{m}\right)$.

1.2. Viscous Profiles. We want to compare the entropy traveling waves with traveling waves of the viscous equation (2). Using the traveling wave ansatz $u(x, t)=$ $u(x-s t)$ in $(2)$, leads to the equation

$$
\varepsilon u^{\prime \prime}=\left(f^{\prime}(u)-s\right) u^{\prime}-g(u)
$$

where the prime denotes differentiation with respect to the new coordinate $\xi:=x-s t$. Note that, unlike in viscous conservation laws, the viscosity parameter $\varepsilon$ is still present in the traveling wave equation.

We can now make precise what we mean by a viscosity traveling wave solution.

Definition 1.3. A traveling wave solution of (1) with wave speed $s_{0}$ is said to be admissible or to admit a viscous profile if there is a sequence of solutions $\left(u_{\varepsilon_{n}}\right)$ of

$$
\varepsilon_{n} u_{\varepsilon_{n}}^{\prime \prime}=\left(f^{\prime}\left(u_{\varepsilon_{n}}\right)-s_{n}\right) u_{\varepsilon_{n}}^{\prime}-g\left(u_{\varepsilon_{n}}\right)
$$

with $\varepsilon_{n} \searrow 0, s_{n} \rightarrow s_{0}$ such that $\left\|u_{\varepsilon_{n}}-u\right\|_{L^{1}(\mathbb{R})} \rightarrow 0$.

Note that this approach is essentially different from Kruzhkov's classical result [Kru70]. While Kruzhkov shows convergence on a finite time interval $[0, T]$ with identical initial data for the hyperbolic and the parabolic equation, we focus on closeness of the profiles for infinite times and allow therefore the initial conditions to differ slightly.

On the other hand, we also allow a small difference in the speeds of the hyperbolic and the viscous wave. This implies that in a fixed frame the $L^{1}$-distance between the two profiles will not remain small.

1.3. The main results. In another article [Här00] we were able to show by classical singular perturbation theory the following statement: 
Proposition 1.1. All heteroclinic waves of type (A) and type (C) admit a viscous profile.

In two cases we had to use different wave speeds $s_{n} \neq s_{0}$. Using the wave speed as an additional parameter is also necessary in order to show that all waves of type (B1) possess a viscous profile, too.

TheOrem 1.1 (Admissibility of type (B1) waves). The continuous traveling wave connecting $u_{\ell}$ to $u_{r}$ is admissible.

More precisely, we will show that for $\varepsilon$ sufficiently small there is a unique value $s(\varepsilon)$ such that a monotone heteroclinic wave $u_{\varepsilon}$ of $(2)$ connects $u_{\ell}$ to $u_{r}$. The wave speed $s(\varepsilon)$ can be shown to depend on the viscosity $\varepsilon$ in the following way:

ThEOREM 1.2 (Asymptotic behavior of the wave speed). For $\varepsilon$ sufficiently small, there is a unique heteroclinic connection $u_{\varepsilon}$ from $u_{\ell}$ to $u_{r}$ that occurs at

$$
s(\varepsilon)=s_{0}-\left.\frac{1}{2} \frac{d}{d u}\left(\frac{g^{\prime}(u)}{f^{\prime \prime}(u)}\right)\right|_{u=u_{m}} \varepsilon+\mathcal{O}\left(\varepsilon^{3 / 2}\right) .
$$

We also present another result which shows that all discontinuous heteroclinic waves do not satisfy the admissibility condition, although the entropy jump condition can be derived from the viscous approximation.

TheOrem 1.3 (Non-admissibility of type (B4) waves). All discontinuous heteroclinic traveling waves with wave speed $f^{\prime}\left(u_{m}\right)$ do not admit a viscous profile.

This will be a consequence of the Jordan curve theorem and thus is probably a low-dimensional effect which shows up only in scalar balance laws.

The rest of this article is organized as follows: In chapter 2 the set-up and some notation is given. The nonexistence theorem 1.3 and the existence theorem 1.1 are proved in chapters 3 and 4, respectively. The final chapter 5 uses a blow-up construction to derive asymptotic estimates for the speed of the viscous traveling waves.

2. Singular perturbation theory. We return now to the viscous traveling wave equation. The second-order equation (6) that arises from plugging the traveling wave ansatz into the parabolic equation (2) can be written as a first-order system in two different ways. Apart from the "phase plane" coordinates

$$
\begin{aligned}
\varepsilon u^{\prime} & =w \\
w^{\prime} & =\frac{\left(f^{\prime}(u)-s\right) w}{\varepsilon}-g(u)
\end{aligned}
$$

one can use the "Liénard coordinates"

$$
\left.\begin{array}{rl}
\varepsilon u^{\prime} & =v+f(u)-s u \\
v^{\prime} & =-g(u)
\end{array}\right\}
$$

We will distinguish these two possibilities by a consequent use of the variable $w$ for phase plane considerations and $v$ for Liénard plane arguments. From the "slow-fast"system (7) two limiting systems can be derived which both capture a part of the behavior that is observed for $\varepsilon>0$.

One is the "slow" system obtained by simply setting $\varepsilon=0$ :

$$
\left.\begin{array}{rl}
0 & =v+f(u)-s u \\
v^{\prime} & =-g(u) .
\end{array}\right\}
$$


The flow is confined to the singular curve

$$
\mathcal{C}_{s}:=\{(u, v): v+f(u)-s u=0\} .
$$

Rescaling the variable $\xi$ we arrive at

$$
\left.\begin{array}{rl}
\dot{u} & =v+f(u)-s u \\
\dot{v} & =-\varepsilon g(u) .
\end{array}\right\}
$$

In the limit $\varepsilon=0$, this equation leads to the "fast" (or "layer") system

$$
\left.\begin{array}{l}
\dot{u}=v+f(u)-s u \\
\dot{v}=0 .
\end{array}\right\}
$$

Equation (9) defines a vector field for which the singular curve $\mathcal{C}_{s}$ consists of equilibrium points only. It points to the left below the curve $\mathcal{C}_{s}$ and to the right above.

Trajectories of the fast system connect only points for which $v+f(u)-s u$ has the same values. This is exactly the Rankine-Hugoniot condition for waves propagating with speed $s$. Moreover the direction of the fast vector field is in accordance with the entropy condition.

The linearization of the fast vector field (9) at an equilibrium $(u,-f(u)+s u)$ possesses one eigenvalue 0 (because there is a one-dimensional manifold of equilibria) and another real eigenvalue $f^{\prime}(u)-s$. We call a branch of $\mathcal{C}_{s}$ stable if $f^{\prime}(u)-s<0$ along this branch and unstable, if $f^{\prime}(u)-s>0$. By assumption (F) for any $s$ there is at most one point where $f^{\prime}(u)=s$. The linearization of (9) at this point which we will call the "fold point" has a double zero eigenvalue, in particular $\mathcal{C}_{s}$ is not normally hyperbolic in a neighborhood of the fold point.

Geometric singular perturbation theory in the spirit of Fenichel [Fen79] is a strong tool to describe trajectories that do not pass through a small neighborhood of this point. Outside such a neighborhood the branches of the singular curve are uniformly normally hyperbolic with respect to the fast field. For this reason, the stable and unstable branches persist as invariant manifolds for $\varepsilon>0$.

Unfortunately, trajectories that pass near the top of the curve $\mathcal{C}_{s}$ are not captured by this classical theory and cannot be avoided in the study of type (B) entropy traveling waves. Such trajectories that switch from a stable branch to an unstable branch and follow the unstable branch for a time of order $\mathcal{O}(1)$ are called canards. They were first described in the Van-der-Pol equation by a group of french nonstandard analysts, see [BCDD81].

3. Nonexistence of viscous profiles. Discontinuous waves of the hyperbolic balance law (1) can be quite complicated. They can possess an arbitrary number of jumps, see [Mas97]. However, all these discontinuous heteroclinic waves from $u_{\ell}$ to $u_{r}$ do not admit a viscous profile.

Proof of Theorem 1.3. We consider a discontinuous heteroclinic traveling wave $u_{0}$ with speed $s_{0}$ parametrized in a way that its leftmost discontinuity is at $\xi=0$. At this first shock $u_{0}$ jumps from $u_{+} \in\left(u_{m}, u_{r}\right)$ to $u_{-} \in\left(u_{\ell}, u_{m}\right)$, see figure 3 . In this figure we have drawn the hyperbolic wave profile as a dashed line projected on the singular curve $\mathcal{C}_{s}$. The shock discontinuity is shown as a horizontal line connecting two points on $\mathcal{C}_{s}$.

For the viscous equation (7) with $\varepsilon$ small and wave speed $s$ near $s_{0}$, the equilibrium $u_{\ell}$ is of saddle type with a one-dimensional unstable manifold. Hence, if there was a 
heteroclinic orbit $u_{\varepsilon}$ from $u_{\ell}$ to $u_{r}$, then it would have to be a branch of the unstable manifold of $u_{\ell}$. We follow thus the unstable manifold of $u_{\ell}$ and show that it cannot converge to $u_{r}$.

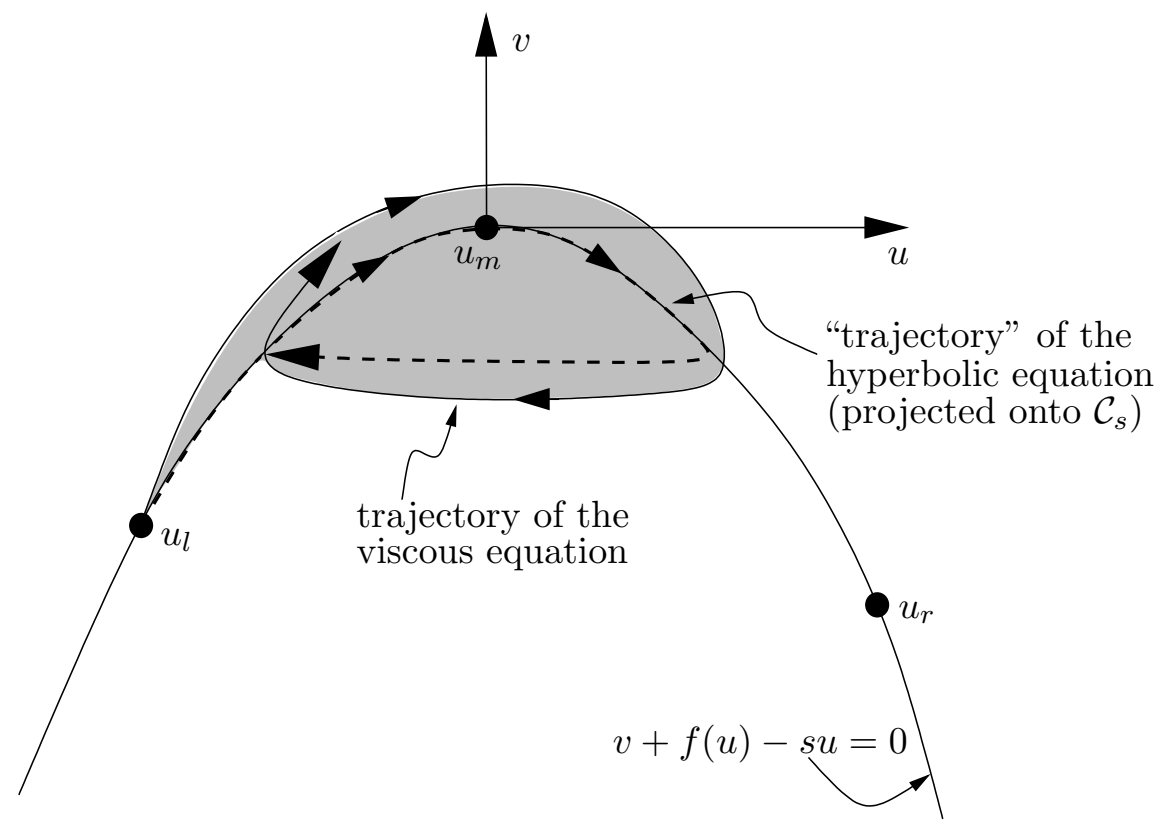

FIG. 1. Why some discontinuous entropy waves cannot possess a viscous profile

If the (smooth) trajectory corresponding to the unstable manifold of $u_{\ell}$ was $L^{1}$ close to $u_{0}$, then it would have to intersect the curve $\mathcal{C}_{s}$ near $u=u_{+}$because $u^{\prime}$ can only change sign by crossing $\mathcal{C}_{s}$. For $\varepsilon$ sufficiently small, the trajectory would then intersect the curve $\mathcal{C}_{s}$ again near $u=u_{-}$and enter a positively invariant region whose boundary is formed by the trajectory and a part of $\mathcal{C}_{s}$. This region is painted in grey in figure 3. Since the grey region is positively invariant and $u_{r}$ lies in the exterior of this region, there can be no heteroclinic connection from $u_{\ell}$ to $u_{r}$ that could provide a viscous profile for the entropy traveling wave $u_{0}$.

4. Admissibility of monotone heteroclinic waves. In this chapter we show that monotone heteroclinic waves of type (B1) do always admit a viscous profile. In a first step we prove, that for fixed $\varepsilon$ small there is a unique wave speed $s(\varepsilon)$ near $s_{0}$ that allows for a heteroclinic connection from $u_{\ell}$ to $u_{r}$ in (7).

4.1. Rotated Vector Fields. A useful concept for the study of some special planar systems are the so-called rotated vector fields introduced by Duff [Duf53] and later improved by Perko [Per75, Per93].

Definition 4.1. Consider a family of planar vector field

$$
\begin{aligned}
& \dot{x}=P(x, y, \nu) \\
& \dot{y}=Q(x, y, \nu)
\end{aligned}
$$

depending on a scalar parameter $\nu$. Let $G: \mathbb{R}^{2} \rightarrow \mathbb{R}$ be an analytic function such that $G(x, y)=0$ defines a curve which is not a trajectory of (10). The family is called a 
family of rotated vector fields $(\bmod \mathbf{G}=0)$ if

$$
\left|\begin{array}{cc}
P(x, y, \nu) & Q(x, y, \nu) \\
P_{\nu}(x, y, \nu) & Q_{\nu}(x, y, \nu)
\end{array}\right| \leq 0 \quad \text { for all } x, y \text { and } \nu
$$

and if the inequality is strict except on the set $\{(x, y) ; G(x, y)=0\}$.

Geometrically, this means that varying the parameter $\nu$, the vector field is "rotated" in the same direction at every point with the possible exception of some points where it may keep its direction. We will use the following

Proposition 4.1 (Duff, Perko). Consider a family of rotated vector fields. Suppose there is an equilibrium, which for all values of $\nu$ possesses a one-dimensional unstable manifold. Then this unstable manifold moves either clockwise or anti-clockwise as the parameter $\nu$ is increased. The stable manifold moves in the same direction. Moreover, these directions are the same for all saddle equilibria of the system.

Lemma 4.1. Consider (7) written in phase plane coordinates

$$
\left.\begin{array}{rl}
\dot{u} & =w / \varepsilon \\
\dot{w} & =\frac{f^{\prime}(u)-s}{\varepsilon} w-g(u)
\end{array}\right\}
$$

depending on the parameters $\varepsilon$ and $s$. Then this is a family of rotated vector fields $(\bmod w=0)$ with respect to the parameter $s$.

Proof. We only have to evaluate (11), i.e.

$$
\left|\begin{array}{ll}
w / \varepsilon & \frac{f^{\prime}(u)-s}{\varepsilon} w-g(u) \\
0 & -w / \varepsilon
\end{array}\right|=-\left(\frac{w}{\varepsilon}\right)^{2} \leq 0
$$

and check that this expression only vanishes on the line $w=0$ which is not a trajectory of the system.

REMARK 1. The vector field in Liénard coordinates is not a rotated vector field. This is the reason why we work here (and only here) in phase plane coordinates.

Now we may use proposition 4.1 to show the existence of a heteroclinic connection from $u_{\ell}$ to $u_{r}$ for the viscous traveling wave equation.

Lemma 4.2. For $\varepsilon$ sufficiently small there exists a unique value $s=s(\varepsilon)$ such that there is a monotone heteroclinic connection $u_{\varepsilon}(\xi)$ from $u_{\ell}$ to $u_{r}$.

Proof. We fix $\varepsilon$ and consider a branch of the unstable manifold $W^{u}\left(u_{\ell}\right)$ and denote with $w_{-}(s)$ the value of its first intersection with the line $u=u_{m}$. In case $W^{u}\left(u_{\ell}\right)$ is a heteroclinic connection between $u_{\ell}$ and $u_{m}$ we set $w_{-}(s):=0$. Similarly the first intersection of $W^{s}\left(u_{r}\right)$ determines a number $w_{+}(s)$. Both functions $w_{-}(s)$ and $w_{+}(s)$ depend continuously on $s$ by standard invariant manifold theory.

Since $s_{0}=f^{\prime}\left(u_{m}\right)$, for $s-s_{0}>2 \sqrt{\varepsilon g^{\prime}\left(u_{m}\right)}$ the equilibrium $u_{m}$ is a sink with two real eigenvalues. In this case the unstable manifold of $u_{\ell}$ connects to $u_{m}$, so $w_{-}(s)=0$. However $w_{+}(s)>0$ since $u_{m}$ is a stable equilibrium and $\dot{w}<0$ along the line $w=0$ as long as $u>u_{m}$. 
For $s-s_{0}<-2 \sqrt{\varepsilon g^{\prime}\left(u_{m}\right)}$ the situation is vice versa: We have $w_{+}(s)=0$ and $w_{-}(s)>0$. The intermediate value theorem yields now immediately the existence of a number $s(\varepsilon)$ for which $w_{-}(s)=w_{+}(s)$. Due to proposition 4.1 we know that the function $w_{-}$is monotone increasing while $w_{+}$is monotone decreasing. From this we can conclude that $s(\varepsilon)$ is unique.

REMARK 2. Since the traveling wave $u_{\varepsilon}$ is monotone in $\xi$, its derivative $u_{\varepsilon}^{\prime}$ is the eigenfunction associated with the first eigenvalue. In turn, this implies that 0 is the largest eigenvalue and hence for each fixed $\varepsilon$ we can conclude that $u_{\varepsilon}$ is linearly stable with respect to perturbations of the viscous balance law (2).

The wave speed $s(\varepsilon)$ of the viscous traveling wave will in general differ from the wave speed $s_{0}$ of the hyperbolic traveling wave. However, they are close to each other as the following lemma shows.

LEMMA 4.3. The speed of the viscous traveling wave is $\mathcal{O}(\varepsilon)$-close to the speed of the hyperbolic traveling wave, i.e. there exists a number $\sigma>0$ such that for $\varepsilon>0$ sufficiently small we have

$$
\left|s(\varepsilon)-s_{0}\right| \leq \sigma \varepsilon
$$

Proof. The argument is purely geometrical. We first define a curve $\gamma$ as a graph

$$
v=\gamma(u)=-f(u)+s u+\frac{\varepsilon g(u)}{f^{\prime}(u)-s_{0}} .
$$

We will show that for $s-s_{0}>\sigma \varepsilon$ the unstable manifold $W^{u}\left(u_{\ell}\right)$ lies below $\gamma$ while the stable manifold $W^{s}\left(u_{r}\right)$ lies above $\gamma$. For $s-s_{0}<-\sigma \varepsilon$ the situation is vice versa: $W^{u}\left(u_{\ell}\right)$ lies above $\gamma$ while $W^{s}\left(u_{r}\right)$ lies underneath. In both cases the manifolds cannot intersect to form a heteroclinic orbit.

We first determine the relative position of $\gamma$ and $W^{u}\left(u_{\ell}\right)$ near $u=u_{\ell}$. The slope of $\gamma$ at $u=u_{\ell}$ is

$$
\gamma^{\prime}\left(u_{\ell}\right)=-f^{\prime}\left(u_{\ell}\right)+s+\frac{\varepsilon g^{\prime}\left(u_{\ell}\right)}{f^{\prime}\left(u_{\ell}\right)-s_{0}} .
$$

The unstable manifold $W^{u}\left(u_{\ell}\right)$ is tangent to the eigenvector $\mathbf{e}_{+}$of the unstable eigenvalue $\lambda_{+}$of the linearization of (7) at the stationary point $u=u_{\ell}, v=-f\left(u_{\ell}\right)+s u_{\ell}$. A straightforward calculation gives

$$
\lambda_{+}=\frac{1}{2 \varepsilon}\left(f^{\prime}\left(u_{\ell}\right)-s+\sqrt{\left(f^{\prime}\left(u_{\ell}\right)-s\right)^{2}-4 \varepsilon g^{\prime}\left(u_{\ell}\right)}\right)
$$

with corresponding eigenvector

$$
\mathbf{e}_{+}=\left(\begin{array}{c}
1 \\
\frac{1}{2} \sqrt{\left(f^{\prime}\left(u_{\ell}\right)-s\right)^{2}-4 \varepsilon g^{\prime}\left(u_{\ell}\right)}-\frac{1}{2}\left(f^{\prime}\left(u_{\ell}\right)-s\right)
\end{array}\right) .
$$

Expanding the square root in powers of $\varepsilon$ we get as the slope of the tangent vector

$$
\begin{aligned}
& f^{\prime}\left(u_{\ell}\right)+s+\frac{\varepsilon g^{\prime}\left(u_{\ell}\right)}{f^{\prime}\left(u_{\ell}\right)-s}+\frac{2 \varepsilon^{2} g^{\prime}\left(u_{\ell}\right)^{2}}{\left(f^{\prime}\left(u_{\ell}\right)-s\right)^{3}}+\mathcal{O}\left(\varepsilon^{3}\right) \\
= & \gamma^{\prime}\left(u_{\ell}\right)+\frac{\varepsilon g^{\prime}\left(u_{\ell}\right)}{f^{\prime}\left(u_{\ell}\right)-s}-\frac{\varepsilon g^{\prime}\left(u_{\ell}\right)}{f^{\prime}\left(u_{\ell}\right)-s_{0}}+\frac{2 \varepsilon^{2} g^{\prime}\left(u_{\ell}\right)^{2}}{\left(f^{\prime}\left(u_{\ell}\right)-s_{0}\right)^{3}}+\mathcal{O}\left(\varepsilon^{3}\right) \\
= & \gamma^{\prime}\left(u_{\ell}\right)+\frac{\varepsilon g^{\prime}\left(u_{\ell}\right)\left(s-s_{0}\right)}{\left(f^{\prime}\left(u_{\ell}\right)-s_{0}\right)\left(f^{\prime}\left(u_{\ell}\right)-s\right)}+\frac{2 \varepsilon^{2} g^{\prime}\left(u_{\ell}\right)^{2}}{\left(f^{\prime}\left(u_{\ell}\right)-s_{0}\right)^{3}}+\mathcal{O}\left(\varepsilon^{3}\right) .
\end{aligned}
$$


Since $g^{\prime}\left(u_{\ell}\right)<0$, this shows that for $s-s_{0}>\sigma \varepsilon$ with $\sigma$ chosen sufficiently large, the unstable manifold lies below $\gamma$ in a right neighborhood of $u=u_{\ell}$.

The same calculation can be carried out near $u=u_{r}$ to show that for $s-s_{0}>\sigma \varepsilon$ the stable manifold $W^{s}\left(u_{r}\right)$ lies above $\gamma$ in a left neighborhood of $u=u_{r}$.

To prove that $W^{u}\left(u_{\ell}\right)$ stays below $\gamma$ we compare the vectorfield (7) along $\gamma$ with the slope $\gamma^{\prime}$. We have

$$
\begin{aligned}
\left.\frac{\dot{v}}{\dot{u}}\right|_{v=\gamma(u)} & =\frac{-\varepsilon g(u)}{\gamma(u)+f(u)-s u} \\
& =-f^{\prime}(u)+s_{0} \\
& =\gamma^{\prime}(u)-\left(s-s_{0}\right)-\varepsilon \frac{\left(f^{\prime}(u)-s_{0}\right) g^{\prime}(u)-f^{\prime \prime}(u) g(u)}{\left(f^{\prime}(u)-s_{0}\right)^{2}} .
\end{aligned}
$$

Since

$$
\lim _{u \rightarrow u_{m}} \frac{\left(f^{\prime}(u)-s_{0}\right) g^{\prime}(u)-f^{\prime \prime}(u) g(u)}{\left(f^{\prime}(u)-s_{0}\right)^{2}}=\frac{f^{\prime \prime}\left(u_{m}\right) g^{\prime \prime}\left(u_{m}\right)-f^{\prime \prime \prime}\left(u_{m}\right) g^{\prime}\left(u_{m}\right)}{2 f^{\prime \prime}\left(u_{m}\right)^{2}}
$$

the last term is uniformly bounded on the interval $\left(u_{\ell}, u_{r}\right)$. So, by choosing $s-s_{0}>\sigma \varepsilon$ with $\sigma$ sufficiently large, the slope of the vector field will be strictly smaller than the slope of the curve $\gamma$, in other words, trajectories do cross $\gamma$ from above.

Therefore the unstable manifold $W^{u}\left(u_{\ell}\right)$ cannot intersect the stable manifold $W^{s}\left(u_{r}\right)$ which lies above $\gamma$.

Since the case $s-s_{0}<-\sigma \varepsilon$ is completely analogous, we omit the details.

Using this estimate on the wave speed we are now able to localize the heteroclinic orbit $u_{\varepsilon}$ in the Liénard plane. We do this in two steps: First, we prove estimates for the part of $u_{\varepsilon}$ which is at least $\mathcal{O}\left(\varepsilon^{1 / 2}\right)$ away from $u_{m}$. In a second step we then show that the passage near $u_{m}$ only takes a time of order $\mathcal{O}\left(\varepsilon^{1 / 2}\right)$. Together, this suffices to prove the admissibility of the monotone heteroclinic wave $u_{0}$.

Lemma 4.4. Let $\sigma$ be as in lemma 4.3 and $\delta>0$ be arbitrary. Then there exists some constants $k>0$ and $\varepsilon_{1}>0$ such that for all $0<\varepsilon \leq \varepsilon_{1}$ and $\left|s-s_{0}\right| \leq \sigma \varepsilon$ the region

$$
U_{+}=\left\{u_{\ell} \leq u \leq u_{m}-\delta \varepsilon^{1 / 2},\left|v+f(u)-s u-\frac{\varepsilon g(u)}{f^{\prime}(u)-s_{0}}\right| \leq k \frac{\varepsilon^{3 / 2} g(u)}{u-u_{m}}\right\}
$$

contains a branch of the unstable manifold of $u_{\ell}$ and trajectories of (7) can leave $U_{+}$ only at the right boundary $u=u_{m}-\delta \varepsilon^{1 / 2}$.

Similarly trajectories may enter a region

$$
U_{-}=\left\{u_{m}+\delta \varepsilon^{1 / 2} \leq u \leq u_{r},\left|v+f(u)-s u-\frac{\varepsilon g(u)}{f^{\prime}(u)-s_{0}}\right| \leq k \frac{\varepsilon^{3 / 2} g(u)}{u-u_{m}}\right\}
$$

containing the stable manifold of $u_{r}$ only at $u=u_{m}+\delta \varepsilon^{1 / 2}$. The situation is depicted in figure 4.1.

Proof. Using $\left|s-s_{0}\right|=\mathcal{O}(\varepsilon)$, we get from (13) the expression

$$
\mathbf{e}_{+}=\left(\begin{array}{c}
1 \\
-\left(f^{\prime}\left(u_{\ell}\right)-s\right)+\varepsilon \frac{g^{\prime}\left(u_{\ell}\right)}{f^{\prime}\left(u_{\ell}\right)-s}+\mathcal{O}\left(\varepsilon^{2}\right)
\end{array}\right)
$$




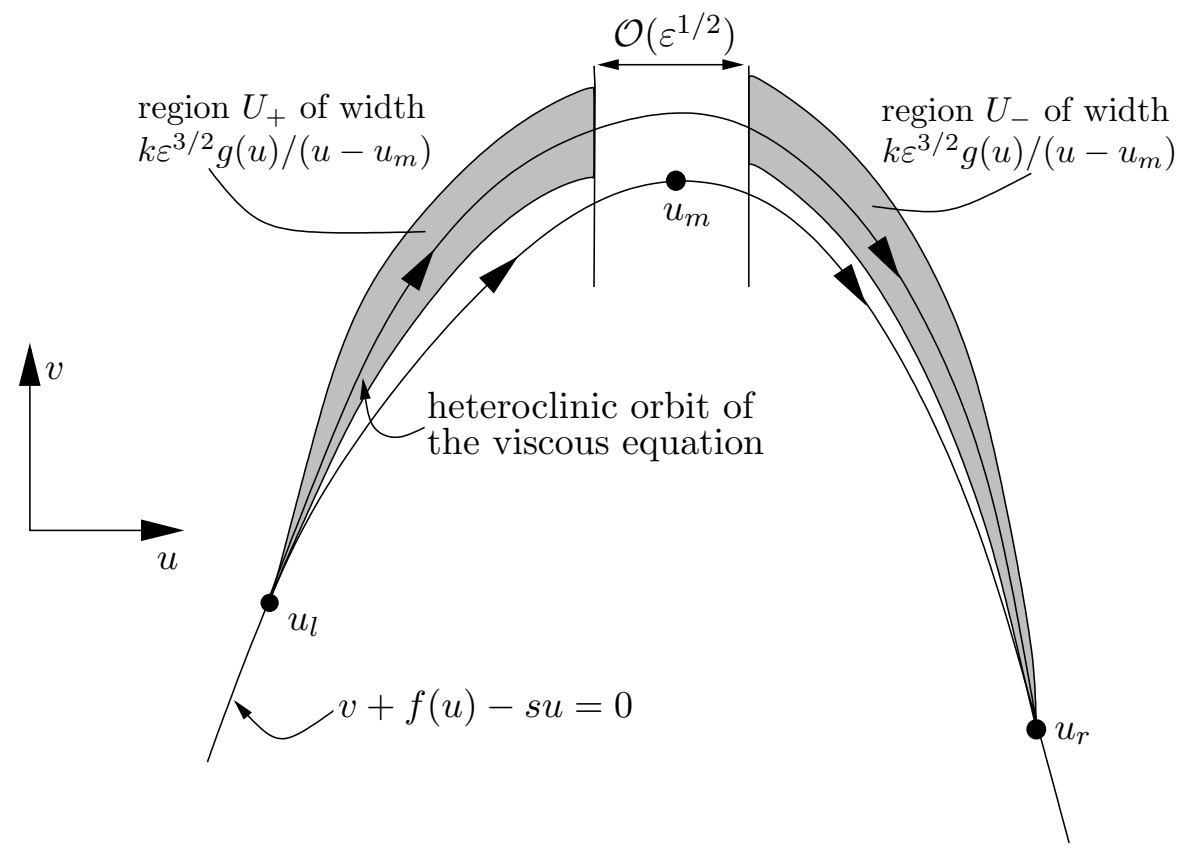

FIG. 2. The heteroclinic orbit at $s=s(\varepsilon)$

for the eigenvector corresponding to the unstable eigenvalue of the linearization of $(7)$ at $u_{\ell}$.

The tangent vectors to the upper and lower boundary of $U_{+}$at $u_{\ell}$ are

$$
\mathbf{t}_{ \pm}=\left(\begin{array}{c}
1 \\
-\left(f^{\prime}\left(u_{\ell}\right)-s\right)+\varepsilon \frac{g^{\prime}\left(u_{\ell}\right)}{f^{\prime}\left(u_{\ell}\right)-s_{0}} \pm k \varepsilon^{3 / 2} \frac{g^{\prime}\left(u_{\ell}\right)}{u_{\ell}-u_{m}}
\end{array}\right)
$$

and by choosing $\varepsilon$ small one can certainly make sure that $\mathbf{e}_{+}$is contained in the open sector between $\mathbf{t}_{-}$and $\mathbf{t}_{+}$. This implies that the unstable manifold of $u_{\ell}$ passes through $U_{+}$.

To show that trajectories leave $U_{+}$only at the right boundary one first considers the upper boundary $v=\bar{\gamma}(u)$ of $U_{+}$where

$$
\bar{\gamma}(u)=-f(u)+s u+\varepsilon \frac{g(u)}{f^{\prime}(u)-s_{0}}+k \varepsilon^{3 / 2} \frac{g(u)}{u-u_{m}} .
$$

The slope of this boundary curve is

$$
\begin{aligned}
\frac{d \bar{\gamma}(u)}{d u}= & -f^{\prime}(u)+s+\varepsilon \frac{\left(f^{\prime}(u)-s_{0}\right) g^{\prime}(u)-f^{\prime \prime}(u) g(u)}{\left(f^{\prime}(u)-s_{0}\right)^{2}} \\
& +k \varepsilon^{3 / 2} \frac{\left(u-u_{m}\right) g^{\prime}(u)-g(u)}{\left(u-u_{m}\right)^{2}} .
\end{aligned}
$$

Since the limits

$$
\lim _{u \rightarrow u_{m}} \frac{\left(f^{\prime}(u)-s_{0}\right) g^{\prime}(u)-f^{\prime \prime}(u) g(u)}{\left(f^{\prime}(u)-s_{0}\right)^{2}}=\frac{f^{\prime \prime}\left(u_{m}\right) g^{\prime \prime}\left(u_{m}\right)-f^{\prime \prime \prime}\left(u_{m}\right) g^{\prime}\left(u_{m}\right)}{2 f^{\prime \prime}\left(u_{m}\right)^{2}}
$$


and

$$
\lim _{u \rightarrow u_{m}} \frac{\left(u-u_{m}\right) g^{\prime}(u)-g(u)}{\left(u-u_{m}\right)^{2}}=-\frac{1}{2} g^{\prime \prime}\left(u_{m}\right)
$$

both exist, it follows that there exists some constant $M>0$ such that

$$
\frac{d \bar{\gamma}(u)}{d u} \geq-f^{\prime}(u)+s-M \varepsilon-M k \varepsilon^{3 / 2} .
$$

On the other hand, the slope of the vector field (7) along the boundary curve $\bar{\gamma}$ of $U_{+}$is

$$
\left.\frac{\dot{v}}{\dot{u}}\right|_{v=\bar{\gamma}(u)}=\frac{-\varepsilon g(u)}{\bar{\gamma}(u)+f(u)-s u}=\frac{-\left(f^{\prime}(u)-s_{0}\right)}{1+\frac{\varepsilon^{1 / 2}\left(f^{\prime}(u)-s_{0}\right)}{u-u_{m}}} .
$$

Using the inequality $(1+\kappa)^{-1}<1-\kappa+\kappa^{2}$ which is valid for $\kappa>0$ this yields

$$
\left.\frac{\dot{v}}{\dot{u}}\right|_{v=\bar{\gamma}(u)} \leq-f^{\prime}(u)+s_{0}+k \varepsilon^{1 / 2} \frac{\left(f^{\prime}(u)-s_{0}\right)^{2}}{u-u_{m}}-k^{2} \varepsilon \frac{\left(f^{\prime}(u)-s_{0}\right)^{3}}{\left(u-u_{m}\right)^{2}}
$$

Let $c_{f}:=\inf _{\left[u_{\ell}, u_{r}\right]} f^{\prime \prime}>0$ and $C_{f}:=\sup _{\left[u_{\ell}, u_{r}\right]} f^{\prime \prime}>0$. For $u \in\left[u_{\ell}, u_{m}-\delta \varepsilon^{1 / 2}\right]$ the mean value theorem shows that

$$
f^{\prime}(u)-s_{0} \leq c_{f}\left(u-u_{m}\right) \leq-c_{f} \delta \varepsilon^{1 / 2} .
$$

Combining this with (14) and (15) one arrives at

$$
\begin{aligned}
& \frac{d \bar{\gamma}(u)}{d u}-\frac{\dot{v}}{\dot{u}} \\
\geq & s-s_{0}-\varepsilon M-k \varepsilon^{3 / 2} M-k \varepsilon^{1 / 2} \frac{f^{\prime}(u)-s_{0}}{u-u_{m}}\left(f^{\prime}(u)-s_{0}\right)+k^{2} \varepsilon \frac{\left(f^{\prime}(u)-s_{0}\right)^{3}}{\left(u-u_{m}\right)^{2}} \\
\geq & -\sigma \varepsilon-\varepsilon M-k \varepsilon^{3 / 2} M+\frac{2}{3} k \varepsilon \delta c_{f}^{2}-\frac{1}{3} k \varepsilon^{1 / 2} c_{f}\left(f^{\prime}(u)-s_{0}\right)+k^{2} \varepsilon \frac{\left(f^{\prime}(u)-s_{0}\right)^{3}}{\left(u-u_{m}\right)^{2}} \\
\geq & \left(-\sigma-M+\frac{1}{3} k \delta c_{f}^{2}\right) \varepsilon+k\left(\frac{1}{3} \varepsilon \delta c_{f}^{2}-\varepsilon^{3 / 2} M\right)+\left(\frac{1}{3} k \varepsilon^{1 / 2} c_{f}-k^{2} \varepsilon C_{f}^{2}\right) \underbrace{\left(s_{0}-f^{\prime}(u)\right)}_{>0} .
\end{aligned}
$$

Taking $k$ sufficiently large such that $\frac{1}{3} k \delta c_{f}^{2}>\sigma+M$ and $\varepsilon$ small we can achieve that $\frac{d \bar{\gamma}(u)}{d u} \geq \frac{\dot{v}}{\dot{u}}$ for $u \in\left[u_{\ell}, u_{m}-\delta \varepsilon^{1 / 2}\right]$. This shows that the vector field along the upper boundary $\bar{\gamma}$ of $U_{+}$points into the interior of $U_{+}$.

In exactly the same way one can show that along the lower boundary the vector field points into the interior of $U_{+}$as well. So the only way how a trajectory can leave $U_{+}$is through the right boundary at $u=u_{m}-\delta \varepsilon^{1 / 2}$.

The proof for the region $U_{-}$is completely analogous and will therefore be omitted. $\square$

Up to now, we know that the heteroclinic orbit $u_{\varepsilon}$ found in lemma 4.2 passes through the regions $U_{+}$and $U_{-}$. This implies that $u_{\varepsilon}^{\prime}$ is $\mathcal{O}\left(\varepsilon^{1 / 2}\right)$-close to the vector field of the hyperbolic traveling wave equation (6) except possibly near the fold point: 
Corollary 4.1. As long as $u_{\varepsilon} \in\left[u_{\ell}, u_{m}-\delta \varepsilon^{1 / 2}\right] \cup\left[u_{m}+\delta \varepsilon^{1 / 2}, u_{r}\right]$ we have

$$
\frac{d u_{\varepsilon}}{d \xi}=\frac{1}{\varepsilon}\left(v_{\varepsilon}+f\left(u_{\varepsilon}\right)-s(\varepsilon) u_{\varepsilon}\right)=\frac{g\left(u_{\varepsilon}\right)}{f^{\prime}\left(u_{\varepsilon}\right)-s_{0}}+\mathcal{O}\left(\varepsilon^{1 / 2}\right) .
$$

Parametrize now the family of heteroclinic orbits $u_{\varepsilon}$ such that $u_{\varepsilon}(0)=u_{m}$ holds for all $\varepsilon$ and fix

$$
\delta_{0}:=\frac{\sqrt{g^{\prime}\left(u_{m}\right)}}{f^{\prime \prime}\left(u_{m}\right)}>0 .
$$

With this parametrization we can find $\xi_{ \pm}=\xi_{ \pm}(\varepsilon)$ such that

$$
u_{\varepsilon}\left(\xi_{ \pm}(\varepsilon)\right)=u_{m} \pm \delta_{0} \sqrt{\varepsilon}
$$

LEMMA 4.5. For $\varepsilon$ sufficiently small and $\xi \in\left[\xi_{-}, \xi_{+}\right]$, we have $u_{\varepsilon}^{\prime}(\xi) \geq c$ with a constant $c>0$ independent of $\varepsilon$. In particular, this implies that $\xi_{+}-\xi_{-}=\mathcal{O}(\sqrt{\varepsilon})$.

Proof. Let $v_{\varepsilon}$ denote the $v$-component of the heteroclinic orbit of (7) which exists at $s=s(\varepsilon)$. According to lemma 4.4, this heteroclinic orbit leaves the strip $U_{+}$at a height

$$
\begin{aligned}
v_{\varepsilon}\left(\xi_{-}\right) & \geq-f\left(u_{\varepsilon}\right)+s(\varepsilon) u_{\varepsilon}\left(\xi_{-}\right)+\frac{\varepsilon g\left(u_{\varepsilon}\right)}{\left(f^{\prime}\left(u_{\varepsilon}\right)-s_{0}\right)}-\frac{k \varepsilon^{3 / 2} g(u)}{u-u_{m}} \\
& =-\frac{\delta_{0}^{2} f^{\prime \prime}\left(u_{m}\right)}{2} \varepsilon+\frac{\varepsilon g^{\prime}\left(u_{m}\right) \sqrt{\varepsilon} \delta_{0}+\mathcal{O}\left(\varepsilon^{2}\right)}{f^{\prime \prime}\left(u_{m}\right) \delta_{0} \sqrt{\varepsilon}+\mathcal{O}(\varepsilon)}+\mathcal{O}\left(\varepsilon^{3 / 2}\right) \\
& =-\frac{\delta_{0}^{2} f^{\prime \prime}\left(u_{m}\right)}{2} \varepsilon+\frac{\varepsilon g^{\prime}\left(u_{m}\right)}{f^{\prime \prime}\left(u_{m}\right)}+\mathcal{O}\left(\varepsilon^{3 / 2}\right) \\
& =\frac{g^{\prime}\left(u_{m}\right)}{2 f^{\prime \prime}\left(u_{m}\right)} \varepsilon+\mathcal{O}\left(\varepsilon^{3 / 2}\right)
\end{aligned}
$$

where lemma 4.3 and the definition of $\delta_{0}$ was used. We can now find $c>0$ such that

$$
v_{\varepsilon}\left(\xi_{-}\right) \geq c \varepsilon
$$

Similarly, $v_{\varepsilon}\left(\xi_{+}\right) \geq c \varepsilon$. Since $v_{\varepsilon}$ is monotone increasing on $\left[\xi_{-}, 0\right]$ and monotone decreasing on $\left[0, \xi_{+}\right]$, we know that

$$
v_{\varepsilon}(\xi)+f\left(u_{\varepsilon}(\xi)\right)-s(\varepsilon) u_{\varepsilon}(\xi) \geq \min \left\{v_{\varepsilon}\left(\xi_{-}\right), v_{\varepsilon}\left(\xi_{+}\right)\right\} \geq c \varepsilon
$$

for $\xi \in\left[\xi_{-}, \xi_{+}\right]$. So, on this small part of the heteroclinic orbit we have indeed

$$
u_{\varepsilon}^{\prime}=\frac{1}{\varepsilon}\left(v_{\varepsilon}+f\left(u_{\varepsilon}\right)-s(\varepsilon) u_{\varepsilon}\right) \geq c .
$$

We are now able to conclude the proof of theorem 1.1. It remains to show that the monotone traveling wave $u_{0}$ of the hyperbolic equation and the viscous heteroclinic waves $u_{\varepsilon}$ with $s=s(\varepsilon)$ satisfy the estimate

$$
\left\|u_{\varepsilon}-u_{0}\right\|_{L^{1}(\mathbb{R})} \rightarrow 0 \text { for } \varepsilon \searrow 0
$$


when they are suitably parametrized.

Proof of theorem 1.1. Recall that the heteroclinic waves $u_{\varepsilon}$ were parametrized according to $u_{\varepsilon}(0)=u_{m}$. Similarly, we fix the parametrization of $u_{0}$ by assuming that $u_{0}(0)=u_{m}$. We need to show that for any given $\rho>0$

$$
\left\|u_{\varepsilon}-u_{0}\right\|_{L^{1}(\mathbb{R})} \leq \rho
$$

holds provided that $\varepsilon$ is sufficiently small. To this end, we split the trajectories in five different parts: For $\xi>\bar{\xi}$ and $\xi<\xi$ with $\bar{\xi}$ and $\xi$ to be determined later, the exponential convergence to $u_{r}$ and $u_{\ell}$ will give us good estimates. For $\xi \in\left[\underline{\xi}, \xi_{-}\right]$and $\xi \in\left[\xi_{+}, \bar{\xi}\right]$ the heteroclinic orbit $\left(u_{\varepsilon}, v_{\varepsilon}\right)$ lies in the regions $U_{+}$and $U_{-}$where we have good control over $u_{\varepsilon}^{\prime}$. By lemma 4.5 , the remaining interval $\left[\xi_{-}, \xi_{+}\right]$is so small that it does not affect the $L^{1}$-estimate:

$$
\int_{\xi_{-}}^{\xi_{+}}\left|u_{\varepsilon}(\xi)-u_{0}(\xi)\right| d \xi=\mathcal{O}(\sqrt{\varepsilon}) \leq \frac{\rho}{5}
$$

for $\varepsilon$ small enough.

To make use of the exponential decay near $u_{\ell}$, we determine $\theta_{\ell}>0$ small such that

$$
\frac{g(u)}{f^{\prime}(u)-s}+k \varepsilon^{1 / 2} \frac{g(u)}{u-u_{m}} \geq \frac{1}{2} \frac{g^{\prime}\left(u_{\ell}\right)\left(u-u_{\ell}\right)}{f^{\prime}\left(u_{\ell}\right)-s_{0}}
$$

for $u_{\ell} \leq u \leq u_{\ell}+\theta_{\ell},\left|s-s_{0}\right| \leq \sigma$ and $0 \leq \varepsilon \leq \varepsilon_{1}$. Moreover, $\theta_{\ell}$ should be so small that

$$
\theta_{\ell} \int_{-\infty}^{0} \exp \left(\frac{g^{\prime}\left(u_{\ell}\right) \xi}{2\left(f^{\prime}\left(u_{\ell}\right)-s_{0}\right)}\right) d \xi \leq \frac{\rho}{10}
$$

Using $\theta_{\ell}$ we can find $\underline{\xi}$ such that $u_{0}(\underline{\xi})<u_{\ell}+\theta_{\ell}$ and $u_{\varepsilon}(\underline{\xi})<u_{\ell}+\theta_{\ell}$ holds for all $\varepsilon$ small enough. This is possible since $u_{\varepsilon}^{\prime}=\frac{g\left(u_{\varepsilon}\right)}{f^{\prime}\left(u_{\varepsilon}\right)-s(\varepsilon)}+\mathcal{O}(\sqrt{\varepsilon})$ is bounded away from zero uniformly in $\varepsilon$ for $u_{\varepsilon} \in\left[u_{\ell}+\theta_{\ell}, u_{m}\right]$.

By corollary 4.1, we have

$$
u_{\varepsilon}^{\prime} \geq \frac{g\left(u_{\varepsilon}\right)}{f^{\prime}\left(u_{\varepsilon}\right)-s(\varepsilon)}+k \varepsilon^{1 / 2} \frac{g\left(u_{\varepsilon}\right)}{u_{\varepsilon}-u_{m}}
$$

for $u_{\varepsilon} \in\left[u_{\ell}, u_{m}-\delta \varepsilon^{1 / 2}\right]$.

Combining this with (17), one gets for $\xi \leq \underline{\xi}$ (where $u_{\varepsilon} \in\left[u_{\ell}, u_{\ell}+\theta_{\ell}\right]$ )

$$
u_{\varepsilon}(\xi)-u_{\ell} \leq \exp \left(\frac{1}{2} \frac{g^{\prime}\left(u_{\ell}\right)(\underline{\xi}-\xi)}{f^{\prime}\left(u_{\ell}\right)-s_{0}}\right)\left(u_{\varepsilon}(\underline{\xi})-u_{\ell}\right) \leq \exp \left(\frac{1}{2} \frac{g^{\prime}\left(u_{\ell}\right)(\underline{\xi}-\xi)}{f^{\prime}\left(u_{\ell}\right)-s_{0}}\right) \theta_{\ell} .
$$

For the same reason, the traveling wave solution $u_{0}$ of the hyperbolic equation satisfies

$$
u_{0}(\xi)-u_{\ell} \leq \exp \left(\frac{1}{2} \frac{g^{\prime}\left(u_{\ell}\right)(\underline{\xi}-\xi)}{f^{\prime}\left(u_{\ell}\right)-s_{0}}\right)\left(u_{0}(\underline{\xi})-u_{\ell}\right) \leq \exp \left(\frac{1}{2} \frac{g^{\prime}\left(u_{\ell}\right)(\underline{\xi}-\xi)}{f^{\prime}\left(u_{\ell}\right)-s_{0}}\right) \theta_{\ell} .
$$

By (18) this implies that

$$
\int_{-\infty}^{\xi}\left|u_{\varepsilon}(\xi)-u_{0}(\xi)\right| d \xi \leq \int_{-\infty}^{\xi}\left(\left|u_{\varepsilon}(\xi)-u_{\ell}\right|+\left|u_{\ell}-u_{0}(\xi)\right|\right) d \xi \leq \frac{\rho}{5} .
$$


In exactly the same way one can show that

$$
\int_{\bar{\xi}}^{+\infty}\left|u_{\varepsilon}(\xi)-u_{0}(\xi)\right| d \xi \leq \frac{\rho}{5} .
$$

Here $\bar{\xi}$ is determined similarly as $\underline{\xi}$ by the conditions $u_{0}(\bar{\xi})>u_{r}-\theta_{r}$ and $u_{0}(\bar{\xi})>u_{r}-\theta_{r}$ for some $\theta_{r}$ satisfying

$$
\theta_{r} \int_{0}^{\infty} \exp \left(\frac{g^{\prime}\left(u_{r}\right) \xi}{2\left(f^{\prime}\left(u_{r}\right)-s_{0}\right)}\right) d \xi \leq \frac{\rho}{10} .
$$

For the estimate on the intermediate interval $\left[\underline{\xi}, \xi_{-}\right]$, we note first that $\mid u_{\varepsilon}\left(\xi_{-}\right)-$ $u_{0}\left(\xi_{-}\right) \mid=\mathcal{O}(\sqrt{\varepsilon})$ by lemma 4.5. Since the heteroclinic trajectory $\left(u_{\varepsilon}, v_{\varepsilon}\right)$ passes through $U_{+}$we have for $\xi \in\left[\underline{\xi}, \xi_{-}\right]$

$$
\begin{aligned}
& \left|u_{\varepsilon}(\xi)-u_{0}(\xi)\right|-\left|u_{\varepsilon}\left(\xi_{-}\right)-u_{0}\left(\xi_{-}\right)\right| \\
\leq & \int_{\xi_{-}}^{\xi}\left|u_{\varepsilon}^{\prime}(\zeta)-u_{0}^{\prime}(\zeta)\right| d \zeta \\
\leq & \int_{\xi_{-}}^{\xi}\left|\frac{g\left(u_{0}\right)}{f^{\prime}\left(u_{0}\right)-s_{0}}-\frac{g\left(u_{\varepsilon}\right)}{f^{\prime}\left(u_{\varepsilon}\right)-s(\varepsilon)}\right|+\mathcal{O}(\sqrt{\varepsilon}) d \zeta \\
= & \int_{\xi_{-}}^{\xi}\left|\frac{g\left(u_{0}\right)}{f^{\prime}\left(u_{0}\right)-s_{0}}-\frac{g\left(u_{\varepsilon}\right)}{f^{\prime}\left(u_{\varepsilon}\right)-s_{0}+\mathcal{O}(\varepsilon)}\right|+\mathcal{O}(\sqrt{\varepsilon}) d \zeta \\
\leq & \int_{\xi_{-}}^{\xi} L\left|u_{\varepsilon}(\zeta)-u_{0}(\zeta)\right| d \zeta+\mathcal{O}(\sqrt{\varepsilon})
\end{aligned}
$$

where $L$ is a Lipschitz constant for the function $g /\left(f^{\prime}-s_{0}\right)$ on $\left[\xi, \xi_{-}\right]$. By the Gronwall inequality, this implies $\left|u_{\varepsilon}(\xi)-u_{0}(\xi)\right|=\mathcal{O}(\sqrt{\varepsilon})$ again for $\xi \in\left[\underline{\xi}, \xi_{-}\right]$. After integration, this yields

$$
\int_{\underline{\xi}}^{\xi_{-}}\left|u_{\varepsilon}(\xi)-u_{0}(\xi)\right| d \xi=\mathcal{O}(\sqrt{\varepsilon}) \leq \frac{\rho}{5}
$$

for $\varepsilon$ small. Exactly by the same reasoning, we get

$$
\int_{\xi_{+}}^{\bar{\xi}}\left|u_{\varepsilon}(\xi)-u_{0}(\xi)\right| d \xi \leq \frac{\rho}{5}
$$

for all sufficiently small $\varepsilon$. Adding up (16) and (19)-(22), we arrive at

$$
\int_{-\infty}^{+\infty}\left|u_{\varepsilon}(\xi)-u_{0}(\xi)\right| d \xi \leq \rho
$$

which completes the proof of theorem 1.1.

5. Asymptotic speed of the viscous traveling wave. In this section we will derive an asymptotic formula for the wave speed of the viscous traveling wave. The tool we use is a blow-up construction similar to the one in [KS01a]. The analysis is very close to the case treated there, however due to violation of a non-degeneracy assumption the "canard case" theorem in [KS01a] does not apply directly. 
Details and background on the blow-up method can be found in [DR96] and [KS01a, KS01b].

From the viewpoint of geometrical singular perturbation theory, parts of the singular curve $\mathcal{C}_{s}$ which are normally hyperbolic, persist for small $\varepsilon>0$. Deleting a small neighborhood of the fold point from $\mathcal{C}_{s}$ leaves two branches: One branch $A_{0}^{s}$ which is attracting for the fast dynamics and one branch $R_{0}^{s}$ which is repelling. By Fenichels theory [Fen79], there will be two invariant curves $A_{\varepsilon}^{s}$ and $R_{\varepsilon}^{s}$ close to $A_{0}^{s}$ and $R_{0}^{s}$ for $\varepsilon>0$ small. In general, these curves are constructed as center manifolds of the slow manifold and hence are not unique.

The dynamics on $A_{\varepsilon}^{s}$ and $R_{\varepsilon}^{s}$ is close to the slow dynamics on $A_{0}$ and $R_{0}^{s}$, in particular, the equilibria $u_{\ell}$ and $u_{r}$, which persist for $\varepsilon>0$, will lie on $A_{\varepsilon}^{s}$ and $R_{\varepsilon}^{s}$. Moreover, $A_{\varepsilon}^{s}$ must contain the unstable manifold of $u_{\ell}$ and $R_{\varepsilon}^{s}$ must contain the onedimensional stable manifold of $u_{r}$, at least up to a vicinity of the fold point. This implies that both $A_{\varepsilon}^{s}$ and $R_{\varepsilon}^{s}$ are determined uniquely. Of course, we may continue $A_{\varepsilon}^{s}$ and $R_{\varepsilon}^{s}$ with the flow in forward, resp. backward direction.

A heteroclinic orbit from $u_{\ell}$ to $u_{r}$ exists if and only if the forward continuation of $A_{\varepsilon}^{s}$ intersects the backward continuation of $R_{\varepsilon}^{s}$. From the previous lemma we know already that such an intersection occurs for precisely one value $s(\varepsilon)$.

This chapter is concerned with the question how to determine, at least to leading order, the wave speed correction $s(\varepsilon)-s_{0}$ caused by the small viscosity.

The key is a good understanding of the dynamics near the fold point when $\varepsilon$ is small and $s$ is varied near $s_{0}$.

To this end, we introduce a new small parameter $\mu$ and rescale the variables according to

$$
\left.\begin{array}{rl}
u & =u_{m}+\mu \bar{u} \\
v & =-f\left(u_{m}\right)+s u_{m}+\mu^{2} \bar{v}, \\
\varepsilon & =\mu^{2} \bar{\varepsilon}
\end{array}\right\}
$$

with

$$
(\bar{u}, \bar{v}, \bar{\varepsilon}) \in S:=\left\{\bar{u}^{2}+\bar{v}^{2}+\bar{\varepsilon}^{2}=1\right\}
$$

It will be convenient to keep the wave speed $s$ as a parameter which will be scaled seperately. The "blow-up" maps the fold point at $\varepsilon=0$ to a sphere $S \times\{\mu=0\}$. Similarly, a full neighborhood of the fold point is mapped to the set $S \times\left[0, \mu_{0}\right)$ and this mapping is one-to-one outside $S \times\{0\}$. As we are interested in values $\varepsilon>0$ we need to study the blown up vector field in a vicinity of the hemisphere $S \cap\{\bar{\varepsilon} \geq 0\}$.

The difference between the usual blow-up of singularities and the approach of [DR96] used here is the fact that the blow up here "mixes" the dynamic variables and the parameters. In particular, $\bar{\varepsilon}$ will in general not be constant along solutions of the rescaled equation. However, the quantity $\mu^{2} \bar{\varepsilon}$ remains a first integral.

To study the flow in the rescaled variables we will change coordinates again and study the blown up vector field in two different sets of coordinates.

The first change of coordinates is given by the transformation

$$
\begin{aligned}
& u_{1}=\bar{u} \bar{\varepsilon}^{-1 / 2} \Rightarrow u=u_{m}+\mu_{1} u_{1} \\
& v_{1}=\bar{v} \bar{\varepsilon}^{-1} \Rightarrow v=-f\left(u_{m}\right)+s u_{m}+\mu_{1}^{2} v_{1} \\
& \mu_{1}=\mu \bar{\varepsilon}^{-1 / 2} \Rightarrow \varepsilon=\mu_{1}^{2} .
\end{aligned}
$$


Note that this change of variables has the same effect as setting $\bar{\varepsilon}=1$ in (23). Moreover, we scale the parameter $s$ as

$$
s-s_{0}=: \mu_{1} s_{1} .
$$

From $\dot{\varepsilon}=0$ we infer $\dot{\mu}_{1}=0$. Hence, in this coordinate system $\mu_{1}$ may also be regarded as a parameter.

In a similar way one can change coordinates for $\bar{v}<0$ according to

$$
\begin{aligned}
& u_{2}=\bar{u}(-\bar{v})^{-1 / 2} \Rightarrow u=u_{m}+\mu_{2} u_{2} \\
& \mu_{2}=\mu(-\bar{v})^{1 / 2} \Rightarrow v=-\mu_{2}^{2}-f\left(u_{m}\right)+s u_{m} \\
& \varepsilon_{2}=-\bar{\varepsilon} \bar{v}^{-1} \Rightarrow \varepsilon=\mu_{2}^{2} \varepsilon_{2}
\end{aligned}
$$

which amounts to the same as setting $\bar{v} \equiv-1$ in (23).

In addition we set

$$
s-s_{0}=: \mu_{2} s_{2} .
$$

For $\bar{\varepsilon}>0$ and $\bar{v}<0$ we may use both sets of variables. In their common domain of definition, the change of variables between the two sets of coordinates is given by

$$
\left.\begin{array}{ll}
u_{1}=\frac{u_{2}}{\sqrt{\varepsilon_{2}}}, & u_{2}=\frac{u_{1}}{\sqrt{-v_{1}}} \\
v_{1}=-\frac{1}{\varepsilon_{2}}, & \varepsilon_{2}=-\frac{1}{v_{1}} \\
\mu_{1}=\mu_{2} \sqrt{\varepsilon_{2}}, & \mu_{2}=\mu_{1} \sqrt{-v_{1}} .
\end{array}\right\}
$$

Expanding $f$ and $g$ in a Taylor series near $u_{1}=0$, the viscous traveling wave equation (7) written in the first set of coordinates reads

$$
\left.\begin{array}{l}
\mu_{1} \dot{u}_{1}=v_{1}+A u_{1}^{2}-s_{1} u_{1}+\mu_{1} B u_{1}^{3}+\mathcal{O}\left(\mu_{1}^{2}\right) \\
\mu_{1} \dot{v}_{1}=-D u_{1}-\mu_{1} E u_{1}^{2}+\mathcal{O}\left(\mu_{1}^{2}\right)
\end{array}\right\}
$$

with $A:=\frac{1}{2} f^{\prime \prime}\left(u_{m}\right), B:=\frac{1}{6} f^{\prime \prime \prime}\left(u_{m}\right), D:=g^{\prime}\left(u_{m}\right)$ and $E:=\frac{1}{2} g^{\prime \prime}\left(u_{m}\right)$. Due to the assumptions $(\mathbf{F})$ and $(\mathbf{G})$ we have $D>0$ and $A>0$.

After rescaling the independent variable, we arrive at the system

$$
\left.\begin{array}{rl}
\dot{u}_{1} & =v_{1}+A u_{1}^{2}-s_{1} u_{1}+\mu_{1} B u_{1}^{3}+\mathcal{O}\left(\mu_{1}^{2}\right) \\
\dot{v}_{1} & =-D u_{1}-\mu_{1} E u_{1}^{2}+\mathcal{O}\left(\mu_{1}^{2}\right)
\end{array}\right\}
$$

which is well-defined for all $\mu_{1}$ and which for $\mu_{1} \neq 0$ possesses the same orbits as (25). For $\mu_{1}=s_{1}=0$ this is a well-known equation in the theory of singular perturbations. It is integrable, more precisely

$$
H\left(u_{1}, v_{1}\right):=\left(v_{1}+A u_{1}^{2}-\frac{D}{2 A}\right) e^{2 A v_{1} / D}
$$

is a conserved quantity. Equation (26) possesses a family of periodic orbits which accumulate onto a special unbounded solution $\gamma_{1}$ corresponding to $H=0$. This special solution can be parametrized as

$$
u_{1}(\tau)=\frac{D}{2 A} \tau, \quad v_{1}(\tau)=\frac{D}{2 A}-\frac{D^{2}}{4 A} \tau^{2}
$$


We will now show that in the coordinates (23) this unbounded orbit corresponds to a heteroclinic orbit $\bar{\gamma}$ connecting two equilibria on the "equator" $\bar{\varepsilon}=0$ of $S$.

From the definition of $u_{1}, v_{1}$ we get immediately

$$
\bar{u}^{2}=\bar{\varepsilon} u_{1}^{2}, \quad \bar{v}^{2}=\bar{\varepsilon}^{2} v_{1}^{2}
$$

Since $\bar{u}^{2}(\tau)+\bar{v}^{2}(\tau)+\bar{\varepsilon}^{2}(\tau)=1$ we get a quadratic equation for $\bar{\varepsilon}(\tau)$ which can be solved to give

$$
\bar{\varepsilon}(\tau)=\frac{2\left(-D^{2} \tau^{2}+\sqrt{\left.64 A^{4}+D^{4} \tau^{4}+4 A^{2} D^{2}\left(D \tau^{2}-2\right)^{2}\right)}\right)}{16 A^{2}+D^{2}\left(D \tau^{2}-2\right)^{2}} .
$$

In particular, we can immediately see that $\bar{\varepsilon}(\tau) \rightarrow 0$ for $\tau \rightarrow \pm \infty$. Moreover,

$$
\begin{aligned}
& \bar{u}(\tau)=\bar{\varepsilon}(\tau)^{1 / 2} \cdot \frac{D}{2 A} \tau \rightarrow \pm \frac{\sqrt{\sqrt{1+4 A^{2}}-1}}{\sqrt{2} A} \\
& \bar{v}(\tau)=\bar{\varepsilon}(\tau) \cdot\left(\frac{D}{2 A}-\frac{D^{2}}{4 A} \tau^{2}\right) \rightarrow \frac{\sqrt{1+4 A^{2}}-1}{2 A}
\end{aligned}
$$

as $\tau \rightarrow \pm \infty$. Since all limits exist, the orbit $\gamma$ is indeed a connecting orbit between two equilibria on $S$.

Below we will show by a Melnikov-like calculation that this heteroclinic orbit persists for $s_{1}=s_{1}\left(\mu_{1}\right)$ providing a connection between the unstable manifold of $u_{\ell}$ and the stable manifold of $u_{r}$.

In the second set of coordinates corresponding to $\bar{v} \equiv-1$ in (23), the equations of motion are more complicated:

From the relation $\mu_{2}^{2}=-v-f\left(u_{m}\right)+s u_{m}$ we get

$$
2 \mu_{2} \dot{\mu}_{2}=-\dot{v}=g(u)=D \mu_{2} u_{2}+E \mu_{2}^{2} u_{2}^{2}+\mathcal{O}\left(\mu_{2}^{3}\right)=: \mu_{2} R\left(\mu_{2}, u_{2}\right) .
$$

From $\dot{\varepsilon}=0$ one concludes that

$$
\mu_{2}^{2} \dot{\varepsilon}_{2}=-\mu_{2} \varepsilon_{2} R\left(\mu_{2}, u_{2}\right) .
$$

Similarly, from the $u$-equation we derive

$$
2 \mu_{2} \varepsilon_{2} \dot{u}_{2}=-2+2 A u_{2}^{2}-2 u_{2} s_{2}+2 B \mu_{2} u_{2}^{3}-\varepsilon_{2} u_{2} R\left(\mu_{2}, u_{2}\right)+\mathcal{O}\left(\mu_{2}^{2}\right) .
$$

Hence, the vector field can (after rescaling by a factor $2 \mu_{2} \varepsilon_{2}$ ) be written as

$$
\left.\begin{array}{rl}
\dot{u}_{2} & =-2+2 A u_{2}^{2}-2 u_{2} s_{2}+2 B \mu_{2} u_{2}^{3}-\varepsilon_{2} u_{2} R\left(\mu_{2}, u_{2}\right)+\mathcal{O}\left(\mu_{2}^{2}\right) \\
\dot{\mu}_{2} & =\varepsilon_{2} \mu_{2} R\left(\mu_{2}, u_{2}\right) \\
\dot{\varepsilon}_{2} & =-2 \varepsilon_{2}^{2} R\left(\mu_{2}, u_{2}\right)
\end{array}\right\}
$$

By (24), in these coordinates the unbounded solution $\gamma$ from the first set of coordinates corresponds to

$$
\varepsilon_{2}(\tau)=\frac{4 A}{D^{2} \tau^{2}-2 D}, \quad u_{2}(\tau)=\frac{D \tau}{\sqrt{A\left(D^{2} \tau^{2}-2 D\right)}}, \quad \mu_{2}=s_{2}=0
$$

and hence converges to $\left(u_{2}, \mu_{2}, \varepsilon_{2}\right)=\left( \pm \frac{1}{\sqrt{A}}, 0,0\right)$ as $\tau \rightarrow \pm \infty$. Due to the rescaling by $2 \mu_{2} \varepsilon_{2}$, (28) is not a solution of (27). 
Apart from the invariant subspace $\left\{\mu_{2}=s_{2}=0\right\}$ containing parts of the heteroclinic orbit $\bar{\gamma}$, equation (27) possesses other invariant subspaces that will help us to describe the dynamics. For any $s_{2}$ fixed, there is an invariant line $\left\{\mu_{2}=\varepsilon_{2}=0\right\}$. Restricting (27) to this line yields the equation

$$
\dot{u}_{2}=-2+2 A u_{2}^{2}-2 u_{2} s_{2} .
$$

For small $s_{2}$, there are two equilibria: An attracting equilibrium $p_{a}\left(s_{2}\right)$ with $u_{2}=$ $-\frac{1}{\sqrt{A}}+\mathcal{O}\left(s_{2}\right)$ and one repelling equilibrium $p_{r}\left(s_{2}\right)$ with $u_{2}=\frac{1}{\sqrt{A}}+\mathcal{O}\left(s_{2}\right)$.

For any fixed $s_{2}$, the invariant subspace $\left\{\mu_{2}=\varepsilon_{2}=0\right\}$ is contained in the invariant subspaces $\left\{\varepsilon_{2}=0\right\}$ and $\left\{\mu_{2}=0\right\}$ which will be studied next.

In the invariant two-dimensional subspace $\left\{\varepsilon_{2}=0\right\}$, equations (27) simplify to

$$
\begin{aligned}
& \dot{u}_{2}=-2+2 A u_{2}^{2}-2 u_{2} s_{2}+2 B \mu_{2} u_{2}^{3}+\mathcal{O}\left(\mu_{2}^{2}\right) \\
& \dot{\mu}_{2}=0 .
\end{aligned}
$$

There exist a line $L_{a}\left(s_{2}\right)=\left\{\left(u_{a}\left(\mu_{2}, s_{2}\right), \mu_{2}\right) ; \mu_{2} \geq 0\right\}$ of equilibria emanating from $p_{a}\left(s_{2}\right)$ with $u_{a}\left(\mu_{2}, s_{2}\right)=-\frac{1}{\sqrt{A}}+\mathcal{O}\left(\left|s_{2}\right|+\mu_{2}\right)$. The linearization in $\left(u_{a}\left(\mu_{2}, s_{2}\right), \mu_{2}\right)$ has one negative eigenvalue $-4 \sqrt{A}+\mathcal{O}\left(\mu_{2}+\left|s_{2}\right|\right) \neq 0$ and one zero eigenvalue. This means that the line of equilibria is normally hyperbolic for all $\left|\mu_{2}\right|$ sufficiently small. A similar normally hyperbolic line $L_{r}\left(s_{2}\right)$ of repelling equilibria emanates from $p_{r}\left(s_{2}\right)$.

From the equations defining the blow-up, one can see that these manifolds $L_{a}\left(s_{2}\right)$ and $L_{r}\left(s_{2}\right)$ correspond to the attracting and repelling parts $A_{0}^{s}$ and $R_{0}^{s}$ of the slow manifold $\mathcal{C}^{s}$ in our original setting before the blow-up. In fact, $L_{a}\left(s_{2}\right)$ and $L_{r}\left(s_{2}\right)$ are the extension of $A_{0}^{s}$ and $R_{0}^{s}$ to $\mu=0$.

The two-dimensional invariant subspace $\left\{\mu_{2}=0\right\}$ also contains $p_{a}\left(s_{2}\right)$ and $p_{r}\left(s_{2}\right)$ but as an easy calculation shows, there are no other equilibria. For fixed $\left|s_{2}\right|$ small, the linearization at $p_{a}\left(s_{2}\right)$ has one non-zero eigenvalue and one zero eigenvalue. Therefore there exists a one-dimensional center manifold $C_{a}\left(s_{2}\right)$ of $p_{a}\left(s_{2}\right)$. For $s_{2}=0$ alias $s=s_{0}$ this center manifold is exactly a branch of the heteroclinic orbit $\bar{\gamma}$ we have already found. Similarly, there exists a one-dimensional center manifold $C_{r}\left(s_{2}\right)$ of $p_{r}\left(s_{2}\right)$ in the plane $\left\{\mu_{2}=0\right\}$.

Now we return to the full phase space of the blown-up vector field, i.e. to equation (27). For $\left|s_{2}\right|$ sufficiently small, the linearization in the equilibrium $p_{a}\left(s_{2}\right)$ possesses a positive eigenvalue and a double zero eigenvalue. From this eigenvalue structure the existence of an invariant manifold follows:

Proposition 5.1. Fix $s_{2}$ and consider the equilibrium $p_{a}^{s}=\left(\frac{1}{\sqrt{A}}, 0,0\right)$ of (27). There exists a family of two-dimensional center manifolds $\mathcal{M}_{a}\left(s_{2}\right)$ of the equilibria $p_{a}\left(s_{2}\right)$ which is attracting. The manifold $\mathcal{M}_{a}\left(s_{2}\right)$ contains the line $L_{a}\left(s_{2}\right)$ of equilibria. Moreover, for $s_{2}=0$ the manifold $\mathcal{M}_{a}(0)$ also contains a piece of the heteroclinic orbit $\bar{\gamma}$.

Analogously, for fixed $s_{2}$ with $\left|s_{2}\right|$ sufficiently small, there exists a two-dimensional repelling center manifold $\mathcal{M}_{r}\left(s_{2}\right)$ near the equilibrium $p_{r}\left(s_{2}\right)$. This manifold contains the line $L_{r}\left(s_{2}\right)$ of equilibria. Again for $s_{2}=0$ the manifold $\mathcal{M}_{r}(0)$ contains a part of $\bar{\gamma}$.

We need to find conditions under which there are trajectories in the blown-up equation with $\mu>0$ connecting a neighborhood of $p_{a}$ with a neighborhood of $p_{r}$. This is necessary in order to have a connection from $A_{\varepsilon}^{s}$ to $R_{\varepsilon}^{s}$. Such a connection will automatically be a heteroclinic orbit in the original system (7). 


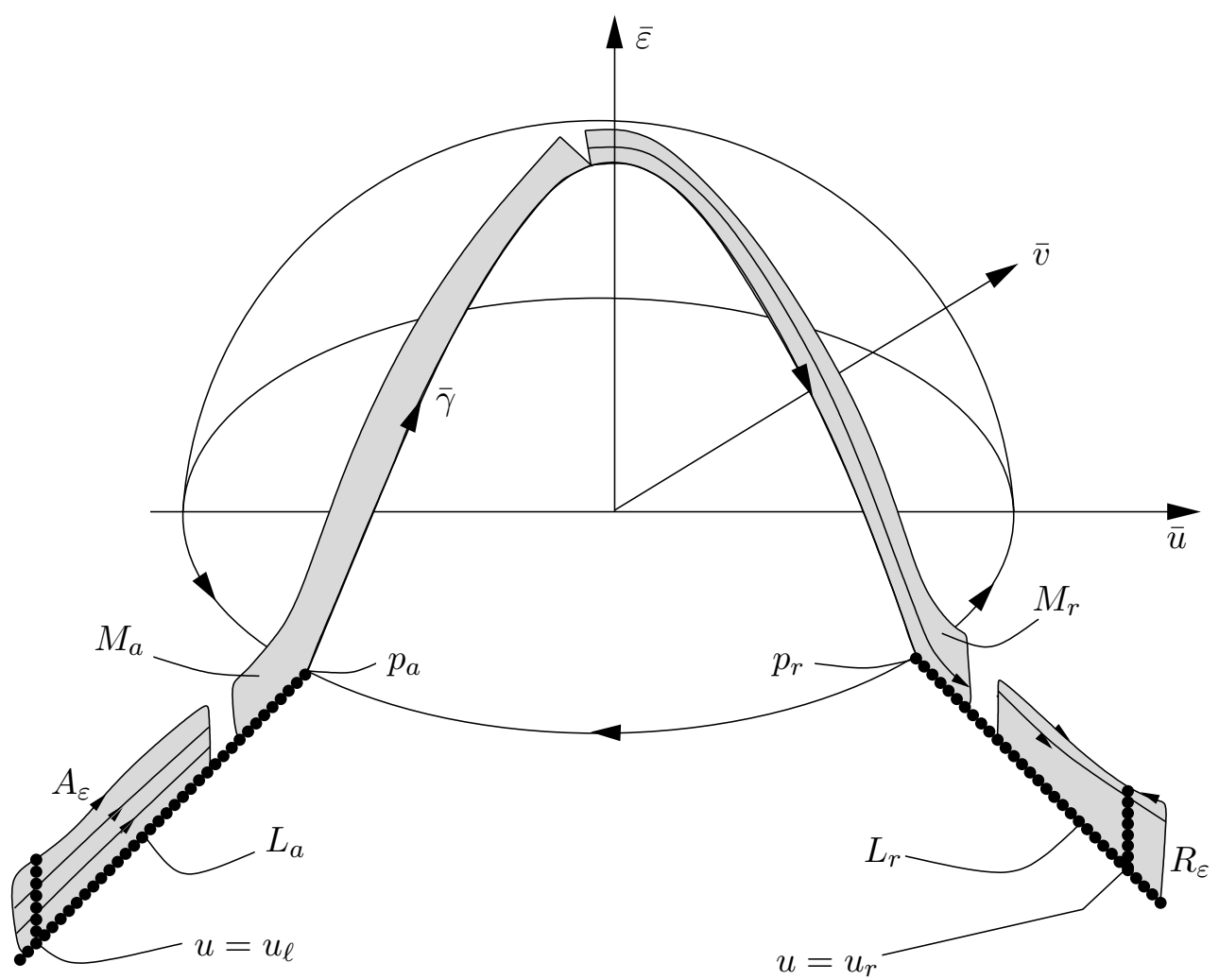

FIG. 3. The dynamics in the blown up vector field for $s=s_{0}$ and the connection to the global heteroclinic orbit

We will therefore determine by a Melnikov-type calculation the relation between $\mu_{2}$ and $s_{2}$ such that an intersection between $\mathcal{M}_{r}\left(s_{2}\right)$ to $\mathcal{M}_{a}\left(s_{2}\right)$ exists. In fact, we know that such a connection exists at $\mu_{2}=s_{2}=0$.

Working in the first set of coordinates again, we apply some recent results of Wechselberger [We02] to determine asymptotically the distance between $\mathcal{M}_{r}$ and $\mathcal{M}_{a}$. To this end, let $d\left(\mu_{1}, s_{1}\right)$ be a distance function measuring the distance of the unstable manifold of $p_{r}$ and the stable manifold of $p_{a}$ at $u_{1}=0$. From the existence of the special solution $\gamma$ we conclude that $d(0,0)=0$. A variant of Melnikov's method can be used to determine the location of the zeroes of $d$ for small nonzero parameters $\mu_{1}$ and $s_{1}$. It has been shown in [KS01a] that the splitting of these manifolds can be measured by the usual Melnikov integrals, although the situation is different from the one typically considered in Melnikov theory: Instead of looking for an intersection of stable and unstable manifolds of two hyperbolic equilibria one looks for the intersection of two noncompact center-stable and center-unstable manifolds associated with unbounded solutions of at most algebraic growth. However, since for this type of solutions the notion of dichotomies is still well defined it is possible to derive Melnikov integrals as a measure for the splitting of these invariant manifolds. For a complete treatment of this situation, see [We02]. We remark that in the second set of coordinates there is only a heteroclinic orbit at $\mu_{2}=0$. It is asymptotic to two non-hyperbolic equilibria which disappear for $\mu_{2} \neq 0$. The Melnikov-like analysis does not yield persistence of a heteroclinic orbit for other values of $\mu_{2}$ in the blown-up 
equations, see figure 5 .

In particular, we may compute $\frac{\partial d}{\partial \mu_{1}}$ and $\frac{\partial d}{\partial s_{1}}$ in the standard way, see [Van92]. To perform the computation one needs explicitly all bounded solutions of the adjoint linearized equation. Linearizing (26) around the solution $\gamma_{1}$ with $\mu_{1}=s_{1}=0$ yields the non-autonomous linear system

$$
\left(\begin{array}{l}
\dot{u} \\
\dot{v}
\end{array}\right)=\left(\begin{array}{cc}
D \tau & 1 \\
-D & 0
\end{array}\right)\left(\begin{array}{l}
u \\
v
\end{array}\right)
$$

The adjoint equation

$$
\dot{\psi}=\left(\begin{array}{ll}
-D \tau & D \\
-1 & 0
\end{array}\right) \psi
$$

has the (up to a constant factor) unique bounded solution

$$
\psi(\tau)=\left(\begin{array}{c}
D \tau e^{-\frac{D}{2} \tau^{2}} \\
e^{-\frac{D}{2} \tau^{2}}
\end{array}\right) .
$$

The Melnikov integral $\frac{\partial d}{\partial \mu_{1}}$ is then computed by integrating the scalar product of $\psi$ with the $\mu_{1}$-derivative of (26) evaluated along the special unbounded solution $\gamma$ :

$$
\begin{aligned}
\frac{\partial d}{\partial \mu_{1}} & =\int_{-\infty}^{+\infty}\left(\begin{array}{c}
D \tau \\
1
\end{array}\right)\left(\begin{array}{c}
\frac{B D^{3}}{8 A^{3}} \tau^{3} \\
-\frac{D^{2} E}{4 A^{2}} \tau^{2}
\end{array}\right) e^{-\frac{D}{2} \tau^{2}} d \tau \\
& =\int_{-\infty}^{+\infty}\left(\frac{B D^{4}}{8 A^{3}} \tau^{4}-\frac{D^{2} E}{4 A^{2}} \tau^{2}\right) e^{-\frac{D}{2} \tau^{2}} d \tau \\
& =\int_{-\infty}^{+\infty}\left(\frac{B D^{3 / 2}}{\sqrt{2} A^{3}} \nu^{4}-\frac{D^{1 / 2} E}{\sqrt{2} A^{2}} \nu^{2}\right) e^{-\nu^{2}} d \nu \\
& =\sqrt{\frac{\pi D}{2}}\left(\frac{3 B D}{4 A^{3}}-\frac{E}{2 A^{2}}\right)
\end{aligned}
$$

Similarly, we find

$$
\begin{aligned}
\frac{\partial d}{\partial s_{1}} & =\int_{-\infty}^{+\infty}\left(\begin{array}{l}
D \tau e^{-\frac{D}{2} \tau^{2}} \\
e^{-\frac{D}{2} \tau^{2}}
\end{array}\right)^{T}\left(\begin{array}{l}
-\frac{D}{2 A} \tau \\
0
\end{array}\right) d \tau \\
& =-\int_{-\infty}^{+\infty} \frac{D^{2}}{2 A} \tau^{2} e^{-\frac{D}{2} \tau^{2}} d \tau \\
& =-\sqrt{\frac{\pi D}{2}} \frac{1}{A} .
\end{aligned}
$$

By the implicit function theorem this implies that $d\left(\mu_{1}, s_{1}\right)=0$ has a solution $s_{1}=$ $s_{1}\left(\mu_{1}\right)$ with

$$
s_{1}\left(\mu_{1}\right)=\left(-\frac{3 B D}{4 A^{2}}+\frac{E}{2 A}\right) \mu_{1}+\mathcal{O}\left(\mu_{1}^{2}\right)
$$


translating this result of the Melnikov calculation back to the original coordinates, we have:

LEMma 5.1. For $\varepsilon$ sufficiently small, there is a unique heteroclinic connection $u_{\varepsilon}$ from $u_{\ell}$ to $u_{r}$ that occurs at

$$
s(\varepsilon)=s_{0}-\left(\frac{f^{\prime \prime \prime}\left(u_{m}\right) g^{\prime}\left(u_{m}\right)-g^{\prime \prime}\left(u_{m}\right) f^{\prime \prime}\left(u_{m}\right)}{2 f^{\prime \prime}\left(u_{m}\right)^{2}}\right) \varepsilon+\mathcal{O}\left(\varepsilon^{3 / 2}\right) .
$$

This concludes the proof of theorem 1.2.

Acknowledgments. This work has been supported by the DFG priority research program ANumE within the project "Viscous profiles". The author thanks P. Szmolyan for bringing his recent work on singular perturbation to his attention and C. Mascia for some helpful comments a long time ago.

\section{REFERENCES}

[BCDD81] E. Benoit, J.-L. Callot, F. Diener and M. Diener, Chasse au canard, Colect. Math., 32(1981), pp. 37-119.

[CG96] G.-Q. Chen AND J. GLImM, Global solutions to the compressible Euler equations with geometrical structure, Comm. Math. Physics, 180(1996), pp. 153-193.

[DR96] F. Dumortier And R. Roussarie, Canard cycles and center manifolds, volume 121 of AMS Memoirs, 1996.

[Duf53] G. F. D. DufF, Limit cycles and rotated vector fields, Ann. Math., 57(1953), pp. 15-31.

[Fen79] N. FENICHEL, Geometric singular perturbation theory for ordinary differential equations, J. Diff. Eq., 31(1979), pp. 53-98.

[Här00] J. HÄRTERICH, Viscous profiles for traveling waves of scalar balance laws: The uniformly hyperbolic case, Electr. J. Diff. Eq., (2000), pp. 1-22, http://ejde.math.swt.edu/Volumes/2000/30/abstr.html.

[KS01a] M. KRUPA AND P. SzMOLYAN, Extending geometric singular perturbation theory to nonhyperbolic points - fold and Canard points in two dimensions, SIAM J. Math. Anal., 33(2001), pp. 266-314.

[KS01b] M. Krupa And P. Szmolyan, Relaxation oscillations and canard explosion, J. Diff. Eq., 174(2001), pp. 312-368.

[Kru70] S. N. KRUZhKov, First order quasilinear equations in several independent variables, Math. USSR Sb., 10(1970), pp. 217-243.

[NT01] P. Noble, S. Travadel. Non-persistence of roll-waves under viscous perturbations, Discr. Cont. Dyn. Syst., Ser. B, 1(2001), pp. 61-70.

[Mas97] C. MAscia, Travelling wave solutions for a balance law, Proc. Roy. Soc. Edinburgh, 127 A(1997), pp. 567-593.

[PC86] V. Roytburd, P. Colella, A. Majda, Theoretical and numerical structure for reacting shock waves, SIAM J. Sci. Stat. Comp., 4(1986), pp. 1059-1080.

[Per75] L. M. Perko, Rotated vector fields and the global behavior of limit cycles of quadratic systems in the plane, J. Diff. Eq., 18(1975), pp. 63-86.

[Per93] L. M. Perko, Rotated vector fields, J. Diff. Eq., 103(1993), pp. 127-145.

[We02] M. WEChSELBERGER, Extending Melnikov theory to invariant manifolds on noncompact domains, Dyn. Syst., 17(2002), pp. 215-233.

[Van92] A. VANDERBAuWhede, Bifurcation of degenerate homoclinics, Results in Mathematics, 21(1992), pp. 211-223. 
